\title{
Influence du type de complément énergétique (rebuts de dattes vs orge) sur les performances d'engraissement et caractéristiques des carcasses d'agneaux Berbères à I'engraissement
}

\author{
Mots-clés \\ Ovin - Date - Croissance - \\ Engraissement - Carcasse - Algérie.
}

\author{
L. Mebirouk-Boudechiche ${ }^{1 *}$ A. Araba ${ }^{2}$ R. Ouzrout ${ }^{1}$
}

\begin{abstract}
Résumé
La valorisation des ressources locales dans les aliments d'animaux en croissance, lorsque celles-ci sont disponibles en quantités appréciables, peut être une opportunité pour réduire les coûts de production et sécuriser le système d'engraissement. Le maintien des performances zootechniques reste toutefois un préalable. Une expérience a porté sur quatre lots de dix agneaux recevant des rations isoprotéiques dans lesquelles des rebuts de dattes ont été incorporés en proportions variables en substitution à l'orge en grain, à raison de $0,15,30$ et 45 p. 100 de la matière sèche de la ration. Les rations ont été distribuées ad libitum par ajustement des quantités offertes. Le régime a eu un effet significatif sur les performances pondérales $(P<0,01)$ et alimentaires $(P<0,001)$. Les gains quotidiens moyens ont augmenté et les indices de consommation ont diminué avec le taux d'incorporation des rebuts de dattes. L'effet du régime s'est aussi révélé significatif sur les rendements en carcasse, mais pas sur le gras de couverture $(P>0,05)$. Les rebuts de dattes peuvent substituer entièrement l'orge en grain dans les rations d'engraissement des ovins sans pour autant affecter leurs performances.
\end{abstract}

\section{INTRODUCTION}

Les rations des agneaux en croissance sont généralement constituées par des mélanges de céréales et d'aliments riches en protéines comme le tourteau de soja, associés à des fourrages frais ou secs. Les céréales constituent un aliment favorable à l'engraissement en raison de l'énergie qu'elles apportent qui est souvent le principal facteur limitant les performances animales.

L'orge, composante de choix de ces céréales, est en Algérie essentiellement importée d'Europe ou d'Amérique. Or, cette céréale, comme le blé, est un produit coté sur les grandes places financières, qui ne cesse de connaître une hausse boursière, accentuée par un taux de change défavorable du dinar. Ce phénomène a pour conséquence de renchérir le prix des concentrés et de pénaliser, de ce fait, les systèmes d'engraissement des ovins à base d'orge. La valorisation de ressources locales, économiques et capables de concurrencer l'orge s'avère une alternative utile.

1. Institut d'Agronomie, Centre universitaire d'El Tarf, BP 73, 36000 El Tarf, Algérie 2. Département des productions animales, Institut agronomique et vétérinaire Hassan II, Rabat-Instituts, Rabat, Maroc.

* Auteur pour la correspondance

E-mail : boudechiche_lamia@yahoo.fr
En Algérie, les rebuts de dattes sont disponibles avec des tonnages appréciables estimés à 67500 t en 2000 (5) et à 100000 t en 2006 (7), pouvant constituer un concentré énergétique alternatif à l'orge pour l'alimentation du bétail local. C'est dans un souci de réduction des coûts de l'alimentation animale et la fourniture de produits de qualité que s'inscrit l'essai rapporté qui vise à étudier l'influence du type de complément énergétique (rebuts de dattes vs orge), par substitution partielle ou totale, sur les performances zootechniques, la consommation alimentaire et la composition corporelle finale des animaux.

\section{MATERIEL ET METHODES}

\section{Animaux, aliments et dispositif expérimental}

Quarante jeunes béliers (7-8 mois) en croissance, de race Berbère, préalablement déparasités et d'un poids vif initial moyen de 31,74 $\mathrm{kg} \pm 0,38$, ont été maintenus dans une étable à stabulation entravée et répartis au hasard en quatre lots de dix animaux chacun. Chaque lot a reçu quotidiennement de la paille $[26$ p. 100 de matière sèche (MS)] et un concentré isoprotéique [18,3 - 19,2 p. 100 de matières azotées totales (MAT)] ad libitum. La composition du concentré a varié selon les lots : 0 p. 100 de rebuts de dattes et 45 p. 100 d'orge pour le lot témoin $0 \%$ R ; 15 p. 100 de rebuts de dattes et 30 p. 100 
d'orge pour le lot $15 \%$ R ; 30 p. 100 de rebuts de dattes et 15 p. 100 d'orge pour le lot $30 \% \mathrm{R} ; 45$ p. 100 de rebuts de dattes et 0 p. 100 d'orge pour le lot 45\%R. Les quatre régimes n'ont différé que par la nature des compléments énergétiques apportés (rebuts de dattes vs orge) et contenaient 1 p. 100 de la MS sous forme de complément minéral vitaminé. Des échantillons représentatifs de chaque ration ont été prélevés pour analyses chimiques selon les méthodes de l'Association of Official Analytical Chemists (3). Les compositions chimiques et alimentaires des rations sont données dans le tableau I.

Après la mise en lots, une période de transition de quinze jours a été ménagée, au cours de laquelle les proportions des régimes expérimentaux ont été progressivement augmentées. L'eau a été disponible à volonté. Les quantités d'aliments offertes et refusées ont été contrôlées quotidiennement par lot. Les animaux ont été pesés individuellement, toutes les deux semaines, à jeun, par double mesure sur deux jours successifs à chaque fois.

A la fin de l'essai, qui a duré 64 jours, six animaux de chaque lot ont été abattus et le poids des carcasses, du gras mésentérique et du gras rénal ont été relevés. L'importance de la graisse à l'extérieur de la carcasse et sur la face interne de la cage thoracique a été appréciée selon les critères de la Communauté économique européenne (4). La fermeté du gras a été déterminée par notation visuelle et tactile en distinguant quatre niveaux de fermeté décrits par le service viande de l'Institut de l'élevage en France (11). Une notation visuelle des dépôts adipeux situés autour de l'attache de la queue et sur le dos de l'animal, basée sur quatre niveaux de coloration (11), a servi de base pour apprécier la couleur du gras de couverture des carcasses d'agneaux.

D'autres mesures ont été effectuées afin de comparer les tissus osseux et musculaire. Le premier a été jugé par le poids des quatre pattes, la longueur de la carcasse et celle de la cuisse, tandis que le tissu musculaire a été jugé par le calcul de l'indice de conformation. Celui-ci est la somme des deux indices suivants :

\section{Tableau I}

Caractéristiques des rations (\% MS)

\begin{tabular}{lcccc} 
& $\mathbf{0} \% \mathbf{R}$ & $\mathbf{1 5} \mathbf{R}$ & $\mathbf{3 0} \% \mathbf{R}$ & $\mathbf{4 5} \mathbf{R}$ \\
\hline Dattes & 0 & 15 & 30 & 45 \\
Orge & 45 & 30 & 15 & 0 \\
$\begin{array}{l}\text { Tourteau } \\
\text { de soja } 48\end{array}$ & 15 & 15 & 15 & 15 \\
Paille & & & & \\
Son de blé & 26 & 26 & 26 & 26 \\
CMV & 13 & 13 & 13 & 13 \\
Phosphate & 1 & 1 & 1 & 1 \\
bicalcique & 0,50 & 0,50 & 0,50 & 0,50 \\
NaCl & & & & \\
Calcaire & 0,40 & 0,40 & 0,40 & 0,40 \\
Composition chimique & & & & 1,40 \\
MS (\%) & 86,25 & 84,82 & 85,11 & 84,20 \\
MO (\%MS) & 92,97 & 93,90 & 93,53 & 94,34 \\
MAT (\%MS) & 19,2 & 18,63 & 18,44 & 18,32 \\
CB (\%MS) & 9,97 & 10,05 & 10,08 & 11,4 \\
MG (\%MS) & 2,3 & 2,4 & 2,4 & 2,4
\end{tabular}

$\mathrm{R}$ : rebuts de dattes ; $\mathrm{CMV}$ : complément minéralisé ou vitaminé ; MS : matière sèche ; MO : matière organique ; MAT : matière azotée totale; $\mathrm{CB}$ : cellulose brute; $\mathrm{MG}$ : matières grasses
Indice de compacité = poids de la carcasse / longueur de la carcasse Indice de muscle = épaisseur de la cuisse / longueur de la cuisse.

La note de conformation a été appréciée d'après une grille codée selon les critères de classement européens (4). Le pH à $24 \mathrm{~h}$ post mortem a été mesuré ainsi que la capacité de rétention en eau sur le Longissimus dorsi par la méthode de Grau et Hamm (1953), cités dans Pearson et Dutson (13).

\section{Analyse statistique}

Toutes les données obtenues ont été soumises à une analyse de variance à un critère (rations) de classification avec le système SAS pour modèle linéaire généralisé (15). Le test de Student-NewmanKeuls (SAS) (15) a permis de rechercher les groupes homogènes de moyennes.

\section{RESULTATS ET DISCUSSION}

\section{Performances zootechniques}

\section{Performances de croissance et d'engraissement}

Les rations ont influencé ( $\mathrm{P} \leq 0,01)$ les poids vifs finaux des agneaux (tableau II), proportionnellement au taux d'incorporation des rebuts de dattes dans la ration (figure 1). Les poids vifs sont passés de $38,95 \mathrm{~kg}$ pour le lot $0 \% \mathrm{R}$ à $40,14 \mathrm{~kg}$ pour le lot $15 \% \mathrm{R}$, et de $40,60 \mathrm{~kg}$ pour le lot $30 \% \mathrm{R}$ à $41,76 \mathrm{~kg}$ pour celui à $45 \% \mathrm{R}$. Ces poids vifs étaient, de ce fait, proportionnels aux taux de rebuts de dattes dans la ration.

La même tendance a été observée pour les gains de poids moyens journaliers (gmq) qui ont été significativement différents entre les lots $(\mathrm{P} \leq 0,01)$. Une amélioration de $44 \mathrm{~g}$ a été observée en faveur du régime $45 \% \mathrm{R}$ par rapport au lot témoin. En conséquence, l'indice de consommation (IC) est passé de près de 15 dans le groupe témoin à moins de 11 dans le groupe $45 \% \mathrm{R}$.

Les références sur l'utilisation des rebuts de dattes chez les ovins manquent. Cependant, les essais conduits sur leur utilisation en tant que concentré énergétique dans la ration d'ovins de race Ouled Djellal, complémentée par de la paille d'orge, ont montré en moyenne des performances pondérales croissantes parallèlement au taux de rebuts de dattes dans la ration, et ceci jusqu'à 75 p. 100 de taux d'incorporation (6).

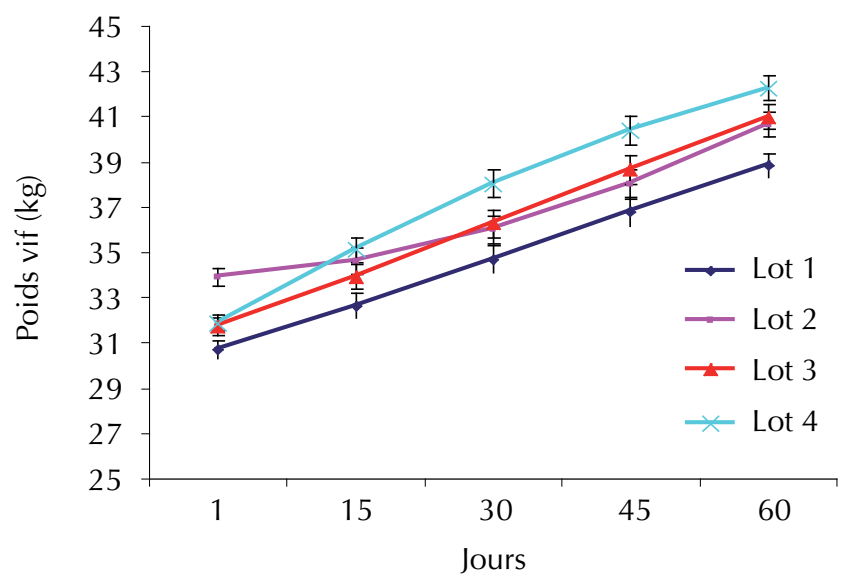

Figure 1 : évolution du poids vif d'agneaux de race Berbère ayant reçu des rations dans lesquelles l'orge a été substituée à des taux de 0 à 45 p. 100 par des rebuts de dattes. 
Il en est de même pour des essais entrepris par Al-Kinani et Alwash (1), et El-Khalisi et coll. (1969), cités par Alwash et DePeters (2), en incorporant des noyaux de dattes à des proportions croissantes dans des rations d'ovins. Chehma et coll. (6) ont rapporté des gmq d'ovins Ouled Djellal, nourris avec une ration contenant 50 p. 100 de rebuts associés à la paille d'orge, inférieurs à ceux des agneaux du lot 45\%R du présent essai (125 g vs $151 \mathrm{~g})$.

Il en résulte que, nonobstant les différentes races utilisées, les performances de croissance sont vraisemblablement meilleures lorsque les rebuts sont mélangés à une source protéique et un complément minéral que lorsqu'ils sont distribués seuls. Les résultats relatifs à cet essai suggèrent l'intérêt de l'utilisation des rebuts de dattes en remplacement de l'orge dans la ration d'engraissement de jeunes agneaux de race Berbère, sans pour autant compromettre les performances de croissance de ces animaux.

\section{Performances de consommation}

Le régime n'a pas eu d'effet sur les performances de consommation $(\mathrm{P}=0,98)$. L'ingestion a été semblable d'un lot à un autre. La consommation journalière moyenne a été proche de $1,58 \mathrm{~kg}$ MS par animal, sur un total distribué de $1,85 \mathrm{~kg}$ de matière sèche par animal (tableau I). Rapportée au poids métabolique, cette consommation a été de 109,30,107,22, 107,75 et 105,84 g MS/kg P $\mathrm{P}^{0,75}$, respectivement pour les lots $0 \% \mathrm{R}, 15 \% 5,30 \% \mathrm{R}$ et $45 \% \mathrm{R}$.

L'IC a été minimal dans le lot $45 \% \mathrm{R}$ avec en moyenne $10,66 \mathrm{~kg}$ d'aliments ingérés par kilogramme de gain de poids vif $(\mathrm{P}<0,05)$. L'effet a été proportionnel au taux d'incorporation des rebuts de dattes. Ces indices de consommation ont été cependant assez élevés, probablement en raison des caractéristiques propres de la race, caractérisée aussi par une croissance lente des agneaux (10).

\section{Caractéristiques des carcasses}

\section{Rendement en carcasse}

Les caractéristiques des carcasses des agneaux sont présentées dans le tableau III. L'effet du régime a été très significatif $(\mathrm{P} \leq 0,01)$ sur les rendements en carcasse, les animaux ayant reçu des rebuts de

\section{Tableau II}

Performances d'engraissement (moyenne \pm ESM) d'agneaux Berbères ayant reçu des rations dans lesquelles de l'orge a été substituée à des taux de 0 à $45 \%$ par des rebuts de dattes $(R)$

\begin{tabular}{|c|c|c|c|c|c|}
\hline & $\mathbf{0} \% \mathbf{R}$ & $15 \% R$ & $30 \% R$ & $45 \% R$ & $P>F$ \\
\hline Nb. d'animaux & 10 & 10 & 10 & 10 & \\
\hline Poids vif initial (kg) & $30,37 \pm 0,38$ & $33,98 \pm 0,38$ & $31,66 \pm 0,37$ & $32,25 \pm 0,39$ & NS \\
\hline Poids vif final (kg) & $38,95^{\mathrm{a}} \pm 0,66$ & $40,14^{b} \pm 0,69$ & $40,60^{b} \pm 0,54$ & $41,76^{b} \pm 0,53$ & $* *$ \\
\hline Durée (jours) & 64 & 64 & 64 & 64 & \\
\hline Gmq (g) & $107,5^{\mathrm{a}} \pm 0,010$ & $126,1^{\mathrm{ab}} \pm 0,011$ & $133,3^{b} \pm 0,008$ & $151,4^{b} \pm 0,008$ & $* *$ \\
\hline Quantité ingérée (g/animal/jour) & $1,58 \pm 0,002$ & $1,57 \pm 0,003$ & $1,59 \pm 0,002$ & $1,58 \pm 0,002$ & NS \\
\hline IC (kg MS/kg) & $14,91^{\mathrm{a}} \pm 1,31$ & $14,28^{b} \pm 1,38$ & $12,62^{b} \pm 1,08$ & $10,66^{b} \pm 1,07$ & * \\
\hline
\end{tabular}

a,b Sur une même ligne, les nombres suivis des mêmes exposants ne diffèrent pas significativement $* \mathrm{P}<0,05 ; * * \mathrm{P}<0,01 ; \mathrm{NS}$ : non significatif

ESM : erreur standard de la moyenne

\section{Tableau III}

Caractéristiques des carcasses d'agneaux de race Berbère ayant reçu des rations dans lesquelles I'orge a été substituée à des taux de 0 à $45 \%$ par des rebuts de dattes

\begin{tabular}{|c|c|c|c|c|c|}
\hline & $\mathbf{0} \% \mathbf{R}$ & $15 \% R$ & $30 \% R$ & $45 \% R$ & $\begin{array}{l}\text { Niveau de } \\
\text { signification }\end{array}$ \\
\hline Nb. d'animaux & 6 & 6 & 6 & 6 & \\
\hline Rendement en carcasse (\%) & $47,36^{\mathrm{a}} \pm 0,63$ & $48,86^{b} \pm 0,45$ & $49,49^{b} \pm 0,45$ & $51,15^{\mathrm{C}} \pm 0,59$ & $* *$ \\
\hline Gras mésentérique (g) & $172,74^{\mathrm{a}} \pm 3,09$ & $300^{b} \pm 2,2$ & $497,51^{\mathrm{c}} \pm 2,22$ & $497,57^{c} \pm 2,9$ & $* * *$ \\
\hline Gras périrénal (g) & $148^{\mathrm{a}} \pm 4,67$ & $176^{b} \pm 3,33$ & $177^{b} \pm 3,36$ & $183^{b} \pm 4,39$ & $* * *$ \\
\hline Etat d'engraissement & 3 & 3 & 3 & 3 & NS \\
\hline Longueur de carcasse $(\mathrm{cm})$ & $79,77^{a} \pm 0,58$ & $77,85^{\mathrm{a}} \pm 0,41$ & $77,83^{\mathrm{a}} \pm 0,42$ & $77,7^{\mathrm{a}} \pm 0,55$ & NS \\
\hline longueur de cuisse (cm) & $45,35^{a} \pm 0,90$ & $43,59^{a} \pm 0,64$ & $43,35^{a} \pm 0,64$ & $44,53^{a} \pm 0,84$ & NS \\
\hline Poids des pattes $(\mathrm{kg})$ & $0,73^{a} \pm 0,018$ & $0,77^{a} \pm 0,013$ & $0,78^{a} \pm 0,013$ & $0,78^{a} \pm 0,017$ & NS \\
\hline Epaisseur des deux cuisses & $18,96^{\mathrm{a}} \pm 0,36$ & $18,93^{\mathrm{a}} \pm 0,36$ & $18,82^{\mathrm{a}} \pm 0,36$ & $18,90^{\mathrm{a}} \pm 0,36$ & NS \\
\hline Indice de compacité & $0,25^{\mathrm{a}} \pm 0,001$ & $0,25^{a} \pm 0,001$ & $0,25^{\mathrm{a}} \pm 0,001$ & $0,25^{\mathrm{a}} \pm 0,001$ & NS \\
\hline Indice de muscle & $0,22^{\mathrm{a}} \pm 0,01$ & $0,21^{\mathrm{a}} \pm 0,01$ & $0,21^{\mathrm{a}} \pm 0,01$ & $0,23^{a} \pm 0,01$ & NS \\
\hline Indice de conformation & $0,47^{a} \pm 0,01$ & $0,46^{a} \pm 0,01$ & $0,46^{\mathrm{a}} \pm 0,01$ & $0,47^{a} \pm 0,01$ & NS \\
\hline
\end{tabular}

a,b,c Sur une même ligne, les nombres suivis des mêmes exposants ne diffèrent pas significativement

$* * \mathrm{P}<0,01 ; * * * \mathrm{P}<0,001 ; \mathrm{NS}:$ non significatif 
datte montrant les valeurs les plus élevées, et ce, proportionnellement aux taux de substitution (tableau III). Farhan et coll. (9) ont également rapporté une amélioration du rendement carcasse à mesure que le niveau de noyaux de dattes augmentait dans la ration.

\section{Importance du tissu adipeux}

A l'abattage, aucune différence n'a été notée entre les régimes en ce qui concerne l'état d'engraissement (gras $=3$ sur une échelle de 1 à 5). Le complément énergétique (rebuts de dattes vs orge) dans la ration n'a donc pas engendré de dépôt plus important de gras de couverture. En revanche, l'augmentation de la proportion des rebuts de dattes dans la ration a engendré des poids de gras de rognon et mésentérique plus importants dans les groupes ayant reçu des rebuts de datte (tableau III), et ce proportionnellement aux taux d'incorporation $(\mathrm{P}<0,001)$.

Ces résultats découleraient de la hiérarchie dans la mise en place des différents dépôts adipeux ; ainsi les dépôts internes et intermusculaires se développent en premier alors que les tissus adipeux sous-cutanés et intramusculaires le font plus tardivement (12). Des résultats similaires ont été enregistrés par Farhan et coll. (9) qui ont observé que le gras de couverture n'était pas affecté par l'augmentation des taux d'incorporation en noyaux de dattes dans les rations. Une épaisseur plus importante du gras caudal (9), une augmentation significative de la surface du muscle Longissimus dorsi, prélevé à la 13 côte d'ovins de race Awassi, et une plus importante épaisseur du gras sous-cutané mesuré au niveau de la même côte ont été rapportées à mesure que le taux en noyaux de dattes augmentait dans la ration (9).

\section{Couleur de carcasse et fermeté du gras}

Aucun défaut de couleur n'a été décelé au niveau des carcasses (appréciées par la couleur du gras de couverture) et la couleur a été identique pour l'ensemble des lots. D'un blanc recouvrant la totalité des carcasses, cette couleur est caractéristique des animaux de bergerie. En effet, l'accumulation dans les graisses de $\beta$-carotènes, pigments colorés contenus dans l'herbe et l'ensilage d'herbe, induit une coloration jaune des gras de couverture (12). La couleur blanche des gras des quatre lots traduirait donc principalement une alimentation à base de concentré constitué majoritairement de céréales pauvres en $\beta$-carotènes.

Tous les gras de couverture analysés 24 h post mortem ont été caractérisés par une mollesse plus ou moins prononcée. Ce défaut de tenue du gras étant quasi entièrement dépendant de l'alimentation animale et de la nature des acides gras s'explique par le fait qu'avec des rations riches en concentré la part de l'acide acétique diminue au profit de l'acide propionique. Celui-ci est ensuite utilisé pour la synthèse d'acides gras saturés impairs à faible point de fusion (12). Par ailleurs, la baisse du $\mathrm{pH}$ diminue l'hydrogénation des lipides polyinsaturés (à faible point de fusion) des aliments qui sont incorporés sous cette forme dans les tissus adipeux. Ces phénomènes expliqueraient l'obtention de gras mous avec des régimes riches en concentré. En revanche, les rations riches en fourrages concourent à l'obtention de gras fermes.

\section{Importance des tissus musculaire et osseux}

L'importance du tissu osseux a été jugée par le poids des pattes, la longueur de la carcasse et celle de la cuisse (tableau III). Aucun de ces paramètres n'a été affecté par le régime $(\mathrm{P}>0,05)$; les moyennes enregistrées ont été de $0,76 \mathrm{~kg}, 78,28 \mathrm{~cm}$ et $44,20 \mathrm{~cm}$, respectivement pour le poids des pattes, la longueur de la carcasse et celle de la cuisse. Le développement du tissu musculaire a été apprécié à partir d'indicateurs rapportés dans le tableau III. Aucune différence n'a été décelée entre les quatre lots en ce qui concerne l'épaisseur de cuisse, l'indice de compacité, l'indice de muscle et celui de conformation (P > 0,05). Le tissu musculaire n'a pas, non plus, été affecté par les régimes.

Cette situation pourrait s'expliquer par le fait que l'orge, qui est une céréale à amidon facilement fermentescible (16), est quasiment dégradée dans le rumen. De ce fait, l'introduction en quantités importantes de glucides facilement fermentescibles dans les rations animales entraîne une orientation des fermentations du rumen qui se traduit par une importante production d'acide propionique, lequel est considéré comme le plus important précurseur de la néoglucogenèse (8), favorisant, entre autres, le métabolisme et la croissance musculaire.

D'autre part, les modèles de fermentation des noyaux de dattes chez les ruminants montre l'accroissement en taux d'acide propionique à mesure que celui en noyaux de dattes augmente dans la ration, et ceci à raison de $0,25,50$ et 75 p. 100 en noyaux (14). Il en résulte que la production de propionates est favorisée non seulement par l'ingestion de l'orge mais aussi par celle des noyaux, ce qui a pour conséquence un développement musculaire identique.

\section{Conformation}

L'appréciation de la conformation, basée sur le développement des profils de la carcasse et notamment des parties essentielles de celle-ci (quartier arrière, dos, épaule), a permis d'attribuer aux quatre lots, une note de conformation, caractérisant un bon développement musculaire, et ne révélant, de ce fait, aucun effet du régime alimentaire sur la conformation des carcasses $(P>0,05)$. Ce résultat est conforme à celui trouvé pour l'indice de conformation, ce qui confirme l'appréciation par notation visuelle.

\section{Qualité de la viande}

Les $\mathrm{pH}$ ultimes ont été normaux avec des valeurs proches de 5,6 (tableau IV). Le régime alimentaire n'a pas eu d'effet sur ce paramètre $(\mathrm{P}>0,05)$. Il en est de même concernant le pouvoir de

\section{Tableau IV}

Caractéristiques de la qualité de la viande d'agneaux Berbères ayant reçu des rations dans lesquelles I'orge a été substituée à des taux de 0 à $45 \%$ par des rebuts de dattes $(R)$

\begin{tabular}{lccrrr} 
& $\mathbf{0} \% \mathbf{R}$ & $\mathbf{1 5} \% \mathbf{R}$ & $\mathbf{3 0} \mathbf{R}$ & $\mathbf{4 5} \mathbf{\%} \mathbf{R}$ & Niveau de signification \\
\hline Nb. d'animaux & 6 & 6 & 6 & 6 & \\
pH & $5,6^{\mathrm{a}} \pm 0,09$ & $5,6^{\mathrm{a}} \pm 0,06$ & $5,7^{\mathrm{a}} \pm 0,06$ & $5,6^{\mathrm{a}} \pm 0,08$ & $\mathrm{NS}$ \\
PRE (\%) & $18,45^{\mathrm{a}} \pm 0,47$ & $18,03^{\mathrm{a}} \pm 0,33$ & $18,34^{\mathrm{a}} \pm 0,34$ & $18,70^{\mathrm{a}} \pm 0,44$ & $\mathrm{NS}$
\end{tabular}

PRE : pouvoir de rétention en eau

a Sur une même ligne, les nombres suivis du même exposant ne diffèrent pas significativement

NS : non significatif 
rétention en eau $(\mathrm{P}>0,05)$. La qualité de la viande appréciée par ces deux paramètres n'a donc pas été influencée par la nature du régime alimentaire.

\section{- CONCLUSION}

Les résultats obtenus montrent que les rebuts de dattes peuvent être incorporés dans les rations de jeunes ovins pour engraissement. En effet, leur emploi en remplacement de l'orge dans les régimes des ovins en croissance a engendré des performances pondérales supérieures ainsi qu'une meilleure efficacité alimentaire. Les trois rations à proportions de rebuts croissants permettent des vitesses de croissance supérieures à celles obtenues avec la ration à base d'orge seule.

Concernant les carcasses produites, un effet significatif a été enregistré sur les rendements au profit du lot $45 \%$ R. En terme d'adiposité, à part les gras de rognon et mésentérique qui ont été plus importants chez les animaux ayant reçu des proportions élevées en rebuts, le gras de couverture a été similaire entre lots, ce qui révèle que, malgré les performances pondérales supérieures des lots ayant reçu des quantités de rebuts croissantes, l'état d'engraissement n'a pas été affecté et est resté en général identique entre lots sans être excessif.

La viande des ovins alimentés à base de rebuts de dattes est apparue d'excellente qualité, identique à celle des ovins alimentés à base d'orge, comme en témoignent les valeurs de $\mathrm{pH}$ à $24 \mathrm{~h}$ et le pouvoir de rétention en eau.

Les rebuts de dattes peuvent être considérés comme un aliment sécurisant, permettant d'atteindre des niveaux de performances élevés et la production de carcasse et de viande de qualité si, toutefois, ils sont complémentés. Ils peuvent donc constituer une source énergétique alternative intéressante en remplacement de l'orge importée et contribuer ainsi à l'autonomie nationale et à la rentabilité des exploitations en Algérie.

\section{BIBLIOGRAPHIE}

1. AL-KINANI L.M.Z., ALWASH A.H., 1975. Study of different proportions of date stones in the ration for fattening Awassi lambs. Iraq J. Agric. Sci., 10: 53-61.

2. ALWASH A.H., DEPETERS E.J., 1982. Date stones for feeding ruminants. World Rev. Anim. Prod., 18: 30-32.

3. AOAC, 1990. Official methods of analysis. Washington, DC, USA, AOAC.

4. CEE, 1992. Règlement $n^{\circ}$ 2137/92, relatif à la grille communautaire de classement des carcasses d'ovins et à la qualité type communautaire des carcasses d'ovins fraîches ou réfrigérées et prorogeant le règlement $n^{\circ}$ 338/91. Bruxelles, Belgique, CEE.

5. CHEHMA A., LONGO H.F., SIBOUKEUR A., 2000. Estimation du tonnage et valeur alimentaire des sous-produits du palmier dattier chez les ovins. Revue Rech. agron. Inraa, 7: 7-15.

6. CHEHMA A., LONGO H.F., SIBOUKEUR A., 2004. Bilan azoté et gain de poids, chez le dromadaire et le mouton, alimentés à base de sous-produits du palmier dattier, de la paille d'orge et du drinn Aristida pungens. Cahiers Agric., $13: 221-226$.

7. CHEHMA A., SENOUSSI A., TERCHA Y., BENGUEGUA S., 2007. Fabrication de blocs multinutritionnels (BMN) à base de sous-produits de palmier dattier et d'urée. In : Coll. international sur les Biotechnologies, Oran, Maroc, 24-25 nov. 2007.

8. DEMIGNE C., YACOUB C., MORAND C., REMESY C., 1988. Les orientations du métabolisme intermédiaire chez les ruminants. Reprod. Nutr. Dév., 28 : 1-17.

9. FARHAN S.M., SHAKIR M.A., EL-KHALISI I., 1969. The use of date stones for feeding and fattening Awassi lambs. Iraq. J. agric. Sci., 4: 86-94.

10. MEYER C., FAYE B., KAREMBE H., POIVEY J.P., 2004. Guide de l'élevage du mouton méditerranéen et tropical. Libourne, France, CEVA Santé animale, p. 19.

11. NORMAND J., BROUARD-JABET S., 2002. Guide pratique. Apprécier la qualité du gras de couverture des carcasses d'agneaux : couleur et fermeté. Paris, France, Institut de l'élevage.

12. NORMAND J., MOEVI I., LUCBERT J., POTTIER E., 2005. Le point sur l'alimentation des bovins et des ovins et la qualité des viandes. Paris, France, Institut de l'élevage.

13. PEARSON A.M., DUTSON T.R., 1953. Quality attributes and their measurement in meat, poultry and fish product. Adv. Meat Res., 9: 138143.

14. RASHID N.H., ALWASH A.H., 1976. The effect of the proportion of date stones in the diet on its digestion and fermentation in the sheep rumen. Iraq J. Agric. Sci., 11: 51.

15. SAS., 1986. SAS systems for linear models. Cary, NC, USA, SAS Institute.

16. THIVEND P., VERMOREL M., 1971. Etude de I'utilisation des amidons par I'agneau en croissance à l'engrais. Ann. Biol. Anim. Bioch. Biophys., $11: 292-294$

Accepté le 05.02.2010 


\section{Summary}

Mebirouk-Boudechiche L., Araba A., Ouzrout R. Influence of the Type of Energy Supplementation (date stones vs Barley) on Fattening Performances and Carcass Characteristics of Fattening Berber Lambs

When local resources are available, their promotion in the feed of growing animals may reduce production costs and secure the fattening system. However, maintaining zootechnical performances remains a precondition. An experiment was carried out on four groups of ten lambs. Each animal received isoproteinic rations containing date stones as substitutes for barley grains in variable proportions at the rate of $0,15,30$ and $45 \%$ of the dry matter. The rations were distributed ad libitum by adjusting the quantities offered. The diet had a significant effect on weight performances $(P<0.01)$ and daily feed intake $(P<0.001)$. The mean daily weight gains increased and consumption indices decreased with the rate of date stone incorporation. The diet effect was also significant for the carcass yield, but not for the fat cover $(P>0.05)$. Date stones can entirely substitute barley grains in rations of fattening sheep without affecting its performances.

Keywords: Sheep - Date - Growth - Fattening - Carcass Algeria.

\section{Resumen}

Mebirouk-Boudechiche L., Araba A., Ouzrout R. Influencia del tipo de complemento energético (retoños de dátil vs cebada) sobre los rendimientos de engorde y características de las carcasas de corderos Berberes durante el engorde

La valorización de los recursos locales en los alimentos de los animales en crecimiento, cuando éstos están disponibles en cantidades importantes, puede ser una oportunidad para reducir los costos de producción y asegurar el sistema de engorde. Sin embargo, el mantenimiento de los rendimientos zootécnicos es una condición previa. El experimento se llevó a cabo en cuatro lotes de diez corderos que recibieron raciones isoproteicas, incluyendo retoños de de dátiles, que se incorporaron en proporciones variables en substitución de la cebada en grano, a razón de 0,15, 30 y 45\% de la materia seca de la ración. Las raciones fueron distribuidas ad libitum, por ajustamiento de las cantidades ofrecidas. El régimen tuvo un efecto significativo sobre los rendimientos ponderales $(P<0,01)$ y alimenticios $(P<0,001)$. Las ganancias cotidianas promedio aumentaron y los índices de consumo disminuyeron con la tasa de incorporación de los retoños de dátiles. El efecto del régimen fue también significativo sobre los rendimientos de la carcasa, pero no sobre la cobertura de grasa $(\mathrm{P}>0.05)$. Los retoños de dátiles pueden substituir enteramente la cebada en grano dentro de las raciones de engorde de los ovinos sin por eso afectar los rendimientos.

Palabras clave: Ovino - Dátil - Crecimiento - Engordo Canal animal - Argelia. 